\title{
Quantitative model-based imaging of mid-infrared radiation from a turbulent nonpremixed jet flame and plume
}

\author{
Brent A. Rankin, ${ }^{\mathrm{a}, \mathrm{b},{ }^{*}}$ Matthias Ihme, ${ }^{\mathrm{c}}$ and Jay P. Gore ${ }^{\mathrm{a}}$ \\ ${ }^{a}$ School of Mechanical Engineering, Purdue University, West Lafayette, IN 47907, USA \\ ${ }^{\mathrm{b}}$ National Research Council, Air Force Research Laboratory, \\ Wright-Patterson AFB, OH 45433, USA \\ ${ }^{c}$ Department of Mechanical Engineering, Stanford University, Stanford, CA 94305, USA \\ *Corresponding Author: \\ Wright-Patterson AFB, OH 45433, USA \\ Email: brent.rankin.ctr@us.af.mil \\ Phone: 937-656-4936 \\ Fax: 937-656-4101 \\ Article Type: Full-length article \\ Shortened Title: Model-based imaging of radiation from a turbulent flame
}

1790 Loop Road

\begin{abstract}
Current understanding of turbulent reacting flows can be improved by novel quantitative comparisons using highly scalable visualization methods based on volume rendering of timedependent mid-infrared intensities in the form of images with multiple view angles. In this work, the effects of radiation and buoyancy in a turbulent nonpremixed flame and plume are studied by quantitatively comparing measured and computed images of the mid-infrared radiation intensity. To this end, a turbulent nonpremixed jet flame (Reynolds number 15,200 and $\mathrm{CH}_{4} / \mathrm{H}_{2} / \mathrm{N}_{2}$ fuel composition) is considered, representing a benchmark flame configuration of the International Workshop on Measurement and Computation of Turbulent Nonpremixed Flames (TNF Workshop). Quantitative images of the radiation intensity from the flame are acquired using a calibrated high-speed mid-infrared camera and band-pass filters. The camera and filters enable time-dependent measurements of radiation from water vapor and carbon dioxide over the entire flame length and beyond. Results of the solution to the radiative transfer equation are
\end{abstract}


rendered in the form of images using scalar values from large eddy simulations (LES) and a narrowband radiation model. Planar images obtained from experiments and simulations for the radiation intensity display qualitatively comparable features, including localized regions of high and low intensity that are characteristic of turbulent flames. The quantitative comparison of the measured and computed temperature profiles and radiation intensities, particularly in the plume region downstream of the stoichiometric flame length, indicate that including radiation heat loss is important even for weakly radiating flames. The results demonstrate that quantitative experimental and model-based imaging of mid-infrared radiation intensity is useful for assessing the results of narrowband radiation and combustion models.

\section{Keywords}

Turbulent nonpremixed flame; flame radiation; buoyancy; large eddy simulations; non-intrusive measurements; infrared imaging 


\section{Nomenclature}

\section{Symbols}

$a_{p} \quad$ Planck mean absorption coefficient

C reaction progress variable

$C_{p} \quad$ specific heat capacity at constant pressure

$c_{0} \quad$ speed of light in a vacuum

$C_{1} \quad$ first radiation constant $\left(2 \pi h c_{0}^{2}\right)$

$C_{2} \quad$ second radiation constant $\left(h c_{0} / k\right)$

$d \quad$ distance between flame axis and camera

$D$ nominal inner diameter of burner

$D_{t} \quad$ substantial derivative $\left(\partial_{t}+u \cdot \nabla\right)$

Fr Froude number $\left(U_{j} / \sqrt{g D}\right)$

$g \quad$ acceleration due to gravity

h Planck's constant

$H \quad$ enthalpy (Eq. 5)

I radiation intensity

$I_{b \lambda} \quad$ blackbody spectral radiation intensity $\left[C_{1} /\left(\lambda^{5}\left(e^{C_{2} / \lambda T}-1\right)\right)\right]$

$k \quad$ Boltzmann's constant

$p \quad$ partial pressure

$\dot{q}_{r} \quad$ radiation heat loss (Eq. 6)

$r \quad$ flame (observed) radial coordinate

$R$ camera (observer) transverse coordinate

Re Reynolds Number $\left(\rho U_{j} D / \mu\right)$

$s \quad$ path length

$T$ temperature $u \quad$ velocity vector

$U_{j} \quad$ mean bulk jet exit velocity

flame (observed) axial coordinate

camera (observer) axial coordinate

$Y \quad$ mass fraction

$Z \quad$ mixture fraction

$\alpha \quad$ molecular diffusivity

$\alpha_{\lambda} \quad$ spectral optics coefficient

$\Delta H_{f}^{0} \quad$ heat of formation

$\theta \quad$ flame (observed) azimuthal coordinate

$\kappa_{\lambda} \quad$ spectral absorption coefficient

$\lambda_{1}, \lambda_{2}$ filter bandwidth wavelength limits

$\mu \quad$ dynamic viscosity

$\rho \quad$ density

$\sigma \quad$ Stefan-Boltzmann constant

$\tau^{\text {res }} \quad$ subgrid turbulent fluxes

$\tau_{\lambda} \quad$ spectral optical thickness (Eq. 8)

$\chi \quad$ scalar dissipation rate

$\dot{\omega} \quad$ chemical reaction rate

\section{Subscripts}

$i \quad$ species $i$

$\infty \quad$ surrounding environment

$\lambda \quad$ wavelength 


\section{Introduction}

Significant progress has been made towards quantifying the importance of radiation transfer in turbulent flames over the past fifteen years. Experimental and computational studies of radiation transfer in non-luminous turbulent nonpremixed and partially premixed jet flames have utilized an extensive range of techniques such as radiation heat flux measurements [1], spectral radiation intensity measurements [2, 3], stochastic time and space series analyses [2, 3], conditional moment closure calculations [4], composition probability density function calculations [5, 6], Monte Carlo simulations [7], and most recently large eddy simulations (LES) [8-10]. Progress on radiation transfer computations for turbulent reacting flows and combustion systems have been the focus of several review articles [11-16].

Significant opportunities exist for improving radiation and combustion models for turbulent nonpremixed and partially premixed flames, building on the progress discussed in the cited references [1-16]. The opportunities are supported partially by emerging imaging technologies (e.g. calibrated high-speed imaging of mid-infrared radiation [17-20]) and advanced simulation capabilities (e.g. LES and direct numerical simulations). Relatively recent advancements in high speed, high resolution planar sensors with sensitivity in relevant mid-infrared spectral wavelengths make it feasible to acquire quantitative images of mid-infrared radiation measurements from carbon dioxide $\left(\mathrm{CO}_{2}\right)$, water vapor $\left(\mathrm{H}_{2} \mathrm{O}\right)$, carbon monoxide $(\mathrm{CO})$, and methane $\left(\mathrm{CH}_{4}\right)$. This capability is utilized in this work to contribute time-dependent imaging measurements of the radiation intensity from a representative turbulent nonpremixed flame with operating conditions defined by the TNF Workshop [21].

The advancements in mid-infrared imaging technologies can be complemented by the development of corresponding quantitative visualization and volume rendering methods for 
displaying three-dimensional time-dependent simulation results in the form of images with consideration of time and length scale that vary over orders of magnitude. Computational flow imaging allows results from theoretical calculations to be displayed in a format that mimics experimental observations [22]. Volume rendering is one technique for creating twodimensional images from three-dimensional discretely sampled computations or measurements [23-26].

The capability to visualize three-dimensional time-dependent results of fire simulations has been advanced by the development of the Fire Dynamics Simulator and Smokeview programs [27]. Smokeview has made the results from fire simulations more accessible by displaying two and three-dimensional temperature and gas species concentration distributions, velocity fields, and particle paths in an effective manner that allows further insights [27]. The rotation of twodimensional scalar profiles about the flame axis coupled with false coloring techniques has been used to compute qualitative visible images of soot containing laminar diffusion flames [28, 29]. These studies demonstrated that the qualitative comparison of measured and computed planar results is a useful tool for assessing soot formation models in luminous regions of laminar flames. The quantitative comparison of measured and computed images of the radiation intensity from bluff-body stabilized laminar diffusion flames revealed good agreement in the size and shape of the flames and important differences in the flame stabilization region, suggesting improvements in the modeling of soot formation and heat losses [17].

The effects of scalar fluctuations on radiation transfer in turbulent flames have been quantified in several computational studies. For example, composition probability density function calculations of partially premixed jet flames demonstrated that including radiation decreased the peak temperature by $60-330 \mathrm{~K}$ with the larger temperature decrease 
corresponding to flames with larger optical thicknesses [5]. Including turbulence radiation interactions (TRI) further decreased the peak temperature by $20-120 \mathrm{~K}$ [5]. Therefore, TRI accounts for approximately one-third of the temperature decrease caused by radiation.

The effects of TRI in LES and the relative importance of subgrid-scale (SGS) and resolvedscale fluctuations have been receiving increased attention over the last few years [9, 10, 30-32]. Some studies have suggested that the SGS radiation emission should be considered, and the SGS radiation absorption can be neglected for homogeneous isotropic turbulence [30, 31]. Evidently, the largest contribution to the filtered radiation emission and absorption terms could be accounted for using resolved scale scalars. LES of nonluminous and luminous turbulent nonpremixed flames have been investigated using filtered density function, photon Monte Carlo, and line-by-line models [10]. The SGS fluctuations contributed more to emission TRI than the resolved scale fluctuations for LES in which approximately $84 \%$ of the turbulence kinetic energy is resolved [10]. Therefore, including a SGS model in LES computations is important for flames in which emission TRI is significant. The contribution of SGS fluctuations to absorption TRI is negligible in comparison to the contribution of the resolved scale fluctuations. Quantitative evaluation of the importance of including a SGS model for emission TRI in laboratory-scale flames and practical combustion systems is an active and important research direction. This work is focused on developing a novel method for quantitatively comparing measurements and simulations of mid-infrared radiation intensities. The lessons learned from the comparisons are expected to support improvement and development of SGS closure models for the description of turbulence radiation interactions.

Turbulent flame experiments that consider several operating conditions and utilize a range of radiation intensity measurements with adequate spatial and temporal resolution are needed to 
assist the computational studies previously described. The development of corresponding novel quantitative image rendering approaches is needed for comparing measurements and computations with consideration of resolved and unresolved time and length scales. Motivated by these considerations, the primary objectives of this work include the following. First, we measure, interpret, and discuss quantitative images of the radiation intensity from a turbulent nonpremixed jet flame to provide important benchmark data for comparison with LES results. Second, we develop techniques for rendering time-dependent and time-averaged quantitative images of the radiation intensity from the turbulent flame using LES scalar results, a narrowband radiation model, and the radiative transfer equation. Third, we qualitatively and quantitatively compare the measured and computed images and discuss the capabilities and limitations of the LES results. Comparing results with and without radiation and with and without buoyancy connects to the objective of demonstrating that the image rendering technique is capable of quantitatively evaluating LES results and serving as a validation tool for radiation and combustion models. Application of the new image rendering technique to a well-studied flame allows for a quantitative evaluation of the method and demonstrates progress towards application to more complex reacting flows (e.g. soot containing flames) and advanced combustion systems.

\section{Experimental Method}

A turbulent nonpremixed jet flame (DLR-A) [2, 3, 18-20, 33-35] from the TNF Workshop [21] is studied. The flame is stabilized on a long tube tapered to a sharp edge with a nominal inner diameter of $8 \mathrm{~mm}$. The fuel is a mixture of methane (22.1\%), hydrogen (33.2\%), and nitrogen (44.7\%). The mean bulk jet exit velocity is $42.2 \mathrm{~m} / \mathrm{s}$, and the jet exit Reynolds number is 15,200 . The burner is surrounded by a co-flow of air at ambient conditions (supplied through 
a $30 \mathrm{~cm}$ by $30 \mathrm{~cm}$ square cross section) with a mean velocity of $0.3 \mathrm{~m} / \mathrm{s}$. The total heat release rate of the flame is $23 \mathrm{~kW}$, and the radiant heat loss fraction is less than $10 \%$ [1].

Quantitative images of the radiation intensity emitted from the turbulent nonpremixed flame are acquired using a calibrated high speed mid-infrared camera with an indium antimonide (InSb) focal plane array (320 by 256 pixels) $[19,20]$. The images of the radiation intensity are acquired over the entire flame and beyond by traversing the burner in the vertical direction. The experimental arrangement and the definition of the coordinate system are illustrated in Figure 1. The flame (observed) coordinate system is defined with an origin located at the center of the burner exit using cylindrical $(x, r, \theta)$ and Cartesian $(x, y, z)$ coordinates. The mid-infrared camera (observer) coordinate system $(X, Y, R)$ is defined with an origin located at $(x, y, z)=(0, d$, 0 ) using Cartesian coordinates where $d(50 \mathrm{~cm})$ is the distance between the flame axis and camera lens. The chord-like paths through the flame are parallel to within less than $9^{\circ}$ based on the camera optics $(25 \mathrm{~mm}, \mathrm{f} / 2.3$ lens) and distance from the camera lens to the flame axis. The optical arrangement results in a spatial resolution of $0.6 \mathrm{~mm}$ at the flame axis and a solid angle of 0.4 milli-steradians (msr) associated with each pixel. Parallel line-of-sight rays can approximate radiation intensity emitted from the flame and incident on each pixel of the focal plane array.

Three band-pass filters $(2.58 \pm 0.03 \mu \mathrm{m}, 2.77 \pm 0.12 \mu \mathrm{m}, 4.34 \pm 0.12 \mu \mathrm{m})$ are used to measure the radiation emitted from $\mathrm{H}_{2} \mathrm{O}$ and $\mathrm{CO}_{2}$. The exposure time $(6-200 \mu \mathrm{s})$ is adjusted to optimize the camera sensitivity depending on the filter used and region of the flame imaged. The sampling frequency $(325-345 \mathrm{~Hz})$ varies by approximately $5 \%$ depending on the exposure time. The mid-infrared camera is calibrated using a high temperature cavity blackbody source placed the same distance from the camera at the flame centerline to account for atmospheric absorption of the flame radiation. The experimental uncertainty is estimated by measuring the 
radiation intensity from the blackbody source on repeated occasions. The experimental uncertainty in the radiation intensity measurements is less than $10 \%$ (95\% confidence).

The radiation intensity measurements acquired with the calibrated high-speed mid-infrared camera are compared quantitatively with fast infrared array spectrometer measurements $[2,3]$ at selected axial locations where data are available $(x / D=20,40$, and 60$)$. The spectrometer measurements are integrated spectrally over the filter bandwidth accounting for transmission losses through the camera lens and filter as well as the detector response.

\section{Computational Methods}

\subsection{Large Eddy Simulation}

The instantaneous reacting flow field of the turbulent nonpremixed flame is computed using a low-Mach number variable-density LES code with an extended flamelet/progress variable (FPV) combustion model $[8,36]$, in which the enthalpy is introduced as an additional state-space variable to account for radiation heat losses. The LES code solves the instantaneous Favrefiltered conservation equations of mass, momentum, mixture fraction, reaction progress variable, and enthalpy. Transport equations for the Favre-filtered quantities and mixture-fraction variance are written as:

$$
\begin{gathered}
\bar{\rho} \tilde{D} \tilde{Z}=\nabla \cdot(\bar{\rho} \tilde{\alpha} \nabla \tilde{Z})+\nabla \cdot \tau_{Z}^{r e s} \\
\bar{\rho} \tilde{D} \tilde{C}=\nabla \cdot(\bar{\rho} \tilde{\alpha} \nabla \tilde{C})+\nabla \cdot \tau_{C}^{r e s}+\bar{\rho} \tilde{\dot{\omega}}_{C}, \\
\bar{\rho} \tilde{D_{t}} \tilde{H}=\nabla \cdot(\bar{\rho} \tilde{\alpha} \nabla \tilde{H})+\nabla \cdot \tau_{H}^{r e s}+\overline{\dot{q}}_{r} \\
\bar{\rho} \tilde{D_{t} Z^{\prime \prime 2}}=\nabla \cdot\left(\bar{\rho} \tilde{\alpha} \nabla \widetilde{Z^{\prime \prime 2}}\right)+\nabla \cdot \tau_{\widetilde{Z^{\prime 2}}}^{r e s}-2 \bar{\rho} \widetilde{u^{\prime \prime} Z^{\prime \prime}} \cdot \nabla \tilde{Z}-\bar{\rho}_{Z}^{r e s}
\end{gathered}
$$


The governing equations are discretized on a staggered grid using second-order accurate finite differencing. A fractional-step method is employed to advance the continuity and momentum equations, and the scalar transport equations are solved semi-implicitly.

The subgrid turbulent fluxes are modeled using the dynamic Smagorinsky procedure, the turbulent scalar flux is modeled by invoking a gradient transport assumption, and the residual scalar dissipation rate is modeled using spectral arguments [37]. The progress variable is represented by a linear combination of major product mass fractions [38], and is defined here as $\mathrm{C}=Y_{\mathrm{CO}_{2}}+Y_{\mathrm{CO}}+Y_{\mathrm{H}_{2} \mathrm{O}}+Y_{\mathrm{H}_{2}}$. The enthalpy (sensible and chemical) is defined as:

$$
H=\int C_{p} d T+\sum_{i=1}^{N} \Delta H_{f, i}^{0} Y_{i}
$$

where $C_{p}$ is the temperature dependent heat capacity of the mixture at constant pressure. The enthalpy transport equation (Eq. 3) assumes unity Lewis number, constant pressure, and negligible viscous dissipation. The enthalpy transport equation (Eq. 3) reduces to a conserved scalar transport equation in the absence of radiation effects. In this work, the radiation heat loss rate is modeled in the optically thin limit $[39,40]$ following the recommendation of the TNF workshop [21]:

$$
\dot{q}_{r}=4 \sigma\left(T^{4}-T_{\infty}^{4}\right) \sum_{i=1}^{4} p_{i} a_{p, i} .
$$

Radiation contributions from four species $\left(\mathrm{CO}_{2}, \mathrm{CO}, \mathrm{H}_{2} \mathrm{O}\right.$, and $\left.\mathrm{CH}_{4}\right)$ are considered in Eq. (6). The corresponding Planck mean absorption coefficients are calculated using a narrowband radiation model (RADCAL) [41], which are expressed as polynomial curves as a function of temperature. This approach eliminates the significant computational penalty associated with 
calculating incident radiation from multiple rays at the cost of relatively small errors for the present weakly radiating flame [42].

In the present application, the Favre-filtered forms of the governing equations are solved, and all filtered thermochemical quantities (i.e. temperature, species composition, density, chemical source terms, and thermo-viscous-diffusive transport properties) are related to the mixture fraction, reaction progress variable, and enthalpy using the FPV combustion model. These thermochemical quantities are obtained from the solution of the unsteady flamelet equations to account for the long time-scale effects of the heat losses. The flamelet lifetime is tracked in terms of the departure of enthalpy from its adiabatic value in the flamelet tabulation. The reader is referred to Refs. [8, 36, 39] for more details on the model formulation. The filtered thermochemical quantities are modeled using a presumed PDF closure model. The PDF of the mixture fraction and progress variable are modeled using beta and Dirac distributions, respectively. The effects of buoyancy appear in an exact manner in the Favre filtered momentum equation. To assess effects of buoyancy on the subgrid scale results, additional computations are performed corresponding to a Froude number of 150.

The Favre-filtered transport equations are solved on a cylindrical computational domain with boundary conditions prescribed to match the experiments. Turbulent inflow conditions for the fuel-stream are obtained from a periodic pipe-flow simulation, and the velocity boundary conditions of the surrounding air co-flow are prescribed from a hyperbolic tangent profile. A convective outflow boundary condition is used at the outlet boundary, and a slip-free boundary condition is used at the radial boundary. The cylindrical domain $(x / D=120$ by $r / D=45$ by $\theta=2 \pi$ ) is discretized non-uniformly (480 by 180 by 64 differential control volumes in the axial, radial, and azimuthal directions, respectively) resulting in approximately 5.53 million grid 
points. The domain size is comparable to that used in previous simulations [36]. To resolve the plume region, a finer mesh-resolution in axial direction has been employed. The grid is concentrated near the central fuel nozzle and the flame centerline, and the mesh is stretched along the axial and radial directions. The minimum and maximum filter widths in the domain are $\Delta_{\min } / D=2.5 \times 10^{-2}$ (shear layer region at the nozzle lip line) and $\Delta_{\max } / D=2$ (outermost control volume at the outflow plane). A grid refinement analysis on a shorter computational domain (extending to $x / D=20$ ) was performed by doubling the resolution in the radial and axial directions. This refinement had no substantial effect on the statistical flow-field quantities.

\subsection{Model-Based Imaging of Radiation Intensity}

Quantitative time-dependent and time-averaged images of the radiation intensity emitted from the turbulent nonpremixed flame are rendered using the LES scalar results, a narrowband radiation model, and the solution to the radiative transfer equation. The flame is discretized using cylindrical coordinates consistent with the spatial resolution of the LES results as illustrated in Fig. 1. The path lengths associated with the parallel lines-of-sight passing through each differential control volume are calculated using geometrical relationships. The radiation intensity emitted from the lines-of-sight through the flame is calculated using a solution to the radiative transfer equation for non-scattering media,

$$
I=\int_{\lambda_{1}}^{\lambda_{2}} \int_{0}^{\tau_{\lambda}} \alpha_{\lambda} I_{b \lambda}\left(\tau_{\lambda}^{*}\right) e^{-\left(\tau_{\lambda}-\tau_{\lambda}^{*}\right)} d \tau_{\lambda}^{*} d \lambda
$$

The incident radiation on the flame from the surroundings is negligible, and the corresponding term is omitted from Eq. (7). The spectral optics coefficient $\left(\alpha_{\lambda}\right)$ is introduced to account for the combined effects of transmission losses through the lens and filter and for the response of the 
focal plane array. At the central wavelength of each band-pass filter, the spectral optics coefficients are approximately $0.6,0.7$, and 0.8 for the $2.58 \pm 0.03 \mu \mathrm{m}, 2.77 \pm 0.12 \mu \mathrm{m}$, and 4.34 $\pm 0.12 \mu \mathrm{m}$ bands, respectively [20]. The spectral coefficient was measured using Fouriertransform infrared (FTIR) spectroscopy and confirmed to be consistent with manufacturer specifications to within $3 \%$. The spectral optical thickness for the flame gases is defined as:

$$
\tau_{\lambda}=\int_{0}^{s} \kappa_{\lambda} d s
$$

The spectral absorption coefficient $\left(\kappa_{\lambda}\right)$ is calculated from the instantaneous computational results of temperature and $\mathrm{CO}_{2}$ and $\mathrm{H}_{2} \mathrm{O}$ mole fractions used in conjunction with a narrowband radiation model (RADCAL) [41] that includes collision and Doppler broadening effects. The spectral absorption coefficient depends in a nonlinear manner on the instantaneous temperature of the absorbing and emitting gases. Turbulence radiation interactions originate in these nonlinearities and lead to the temperature sensitivity as discussed later in the paper.

The radiation intensity is calculated by integrating along lines-of-sight through the flame, applying the spectral response of the optics and camera, and integrating over the spectral range of the filter to allow for quantitative comparison with the measurements. An instantaneous image of the radiation intensity is rendered by performing the calculations along multiple linesof-sight through the flame consistent with the spatial and temporal resolution of the LES results. The instantaneous model-based mid-infrared images utilize the resolved LES solution for the species concentrations and temperatures. The influence of the subgrid scale species concentration and temperature fluctuations on the local and path integrated radiation intensities can be estimated using the present comparison between computed and measured instantaneous and average images. A time-averaged image of the radiation intensity is obtained by averaging 
200 individual image realizations which are sufficient to obtain statistically converged average image.

\section{Results and Discussion}

\subsection{Large Eddy Simulation Results}

Measured and computed temperature, $\mathrm{CO}_{2}$ concentration, and $\mathrm{H}_{2} \mathrm{O}$ concentration profiles are reported and discussed to assist with quantitatively interpreting the measured and computed radiation intensities. Figure 2 depicts a comparison of the measured [21] and computed mean and root mean square scalar profiles along the flame centerline. The LES results are shown for three cases which exclude radiation (i.e. adiabatic), include radiation (i.e. radiation), and include both radiation and buoyancy (i.e. radiation + buoyancy). The measurements and the three computations are in very good agreement (i.e. to within $50 \mathrm{~K}$ ) along the flame centerline upstream of the stoichiometric flame length (64 diameters). Near and downstream of the stoichiometric flame length, the adiabatic computations over-estimate the mean temperature by as much as $250 \mathrm{~K}$ with the difference increasing with distance downstream. Including the effects of radiation improves the agreement by reducing the computed temperatures by approximately $100 \mathrm{~K}$ along the flame centerline downstream of the stoichiometric flame length. Including the effects of buoyancy improves agreement to a lesser extent by reducing the computed temperature further by approximately $50 \mathrm{~K}$ along the flame centerline downstream of the stoichiometric flame length. The results demonstrate that including buoyancy effects is important in the plume region, and including radiation heat loss is important even for weakly radiating flames (e.g. radiant heat loss fraction less than 10\%). 
Figure 3 compares measured [21] and computed mean and root mean square radial scalar profiles at representative selected axial locations $(x / D=20,40,60$, and 80$)$. The measured and computed mean radial temperature profiles are in excellent agreement at $x / D=20$. At locations further downstream $(x / D=40,60$, and 80$)$, the computations over-estimate the mean temperature by up to $150-200 \mathrm{~K}$ at locations away from the flame axis even when including the effects of radiation and buoyancy. It will be shown subsequently that these differences away from the flame centerline have a significant effect on the path-integrated radiation intensities. The measured and computed mean $\mathrm{CO}_{2}$ and $\mathrm{H}_{2} \mathrm{O}$ concentration radial profiles are in good agreement to within $20 \%$ at locations upstream of the stoichiometric flame length. Farther downstream, the adiabatic computations over-predict mean $\mathrm{CO}_{2}$ and $\mathrm{H}_{2} \mathrm{O}$ concentrations by up to $30 \%$. The inclusion of radiation and buoyancy leads to an improved agreement to within $20 \%$ between the measured and computed $\mathrm{CO}_{2}$ and $\mathrm{H}_{2} \mathrm{O}$ concentrations for the locations downstream of the stoichiometric flame length. The results comparing measured and computed mean and root mean square scalar profiles demonstrate the consistency between conclusions based on the past comparisons of scalar profiles and those based on the present comparisons of path-integrated radiation intensities.

\subsection{Quantitative Model-Based Imaging Results}

Figure 4 shows representative time-dependent and time-averaged images of the radiation intensity from the turbulent nonpremixed flame. Measured images are shown in panels (a) - (c), computed images based on adiabatic LES results are shown in panels (d) - (f), computed images based on LES results which include radiation heat loss are shown in panels (g) - (i), and computed images based on LES results which include both radiation heat loss and buoyancy are 
shown in panels $(\mathrm{j})$ - $(\mathrm{k})$. The time between each of the measured and computed instantaneous images is $3 \mathrm{~ms}$ and $2 \mathrm{~ms}$, respectively. The horizontal white lines, shown in the images, indicate the field of view associated with the measured images. The images are acquired and rendered using a band-pass filter $(2.77 \pm 0.12 \mu \mathrm{m})$ which transmits radiation from $\mathrm{H}_{2} \mathrm{O}$ and $\mathrm{CO}_{2}$. Images acquired and rendered using band-pass filters which transmit radiation only from $\mathrm{H}_{2} \mathrm{O}(2.58 \pm$ $0.03 \mu \mathrm{m})$ or $\mathrm{CO}_{2}(4.34 \pm 0.10 \mu \mathrm{m})$ are qualitatively similar to those reported here.

The computed time-dependent images of the radiation intensity reveal qualitative features that are remarkably similar to those observed in the measured images. A relatively low intensity region is apparent near the burner exit where the line-of-sight paths through the flame are shorter and the path-integrated temperatures are lower. Localized regions of high and low intensity are evident with increasing distance downstream. This is characteristic for the mixing between the fuel jet, entrained air, and combustion products. A wrinkled lower intensity region is observed in the shear layer. The shear layer region becomes increasingly wrinkled with downstream distance as mixing between the exhaust products and the surrounding air becomes more intermittent. In the plume region, the entrained air decreases the temperature and species concentrations, resulting in a rapid decrease in the radiation intensity. The qualitative similarities between the measured and computed results demonstrate the potential of utilizing model-based imaging of radiation intensity for the assessment of radiation and combustion models. The comparisons of radiation intensity measurements and computations complement comparisons of temperature and species measurements and computations by providing two-dimensional multi-ray performance information for model improvement.

To allow for a quantitative interpretation of the results, Fig. 5 shows the measured and computed mean radiation intensities along the image centerline (i.e. diametric paths through the 
flame) for each of the three spectral bands. Table 1 lists the peak mean radiation intensities and the associated relative percent difference between the measured and computed intensities. The measured and computed mean radiation intensities are in good agreement between the burner exit and 20 diameters downstream. Near and downstream of the stoichiometric flame length, the model-based imaging approach over-predicts the mean radiation. The peak mean radiation intensities are over-predicted by approximately 45-60\% (depending on the spectral band) when using adiabatic LES results. Including the effects of radiation heat loss in the LES improves agreement such that the measured and computed radiation intensities agree to within approximately $35-45 \%$ (depending on the spectral band). Including the effects of buoyancy improves the agreement further but only at locations downstream of the stoichiometric flame length. For comparison purposes, it is noted that the differences between the measured and computed radiation intensities for the turbulent diffusion flame (35-45\%) considered in the present work are larger than those observed for bluff-body stabilized laminar diffusion flames (15-30\%) studied in past work [17]. Both the present and past [17] studies utilized similar experimental methods with identical calibration processes and similar model-based mid-infrared imaging approaches with identical narrowband radiation models.

Figure 5 also shows that the mid-infrared camera and spectrometer measurements agree to within the experimental uncertainty of the two instruments, demonstrating the quantitative nature of the present imaging technique. Last, Fig. 5 includes mean radiation intensities at four axial locations $(X / D=20,40,60$, and 80$)$ calculated with a further adjustment such that the computed mean radial temperature profiles are matched to the experimental data. This separate calculation is intended: (a) to assess the extent to which differences between the measured and computed radiation intensities result from models within the LES and the narrowband radiation 
calculations, (b) to illustrate the pronounced sensitivity of the radiation intensities to small differences in the temperature, and (c) to demonstrate the capability of the model-based imaging approach to detect these differences. Table 1 lists measured and computed peak mean radiation intensities (which occur near $X / D=60$ ) for the three spectral bands and the relative percent difference between the measured and the computed values. This location has been selected only for illustration purposes. Comparisons based on values at $X / D=20$ and $X / D=40$ show smaller difference while those at $X / D=80$ show differences that are comparable to the ones at $X / D=60$ for all methods of scalar computation. Results from the adjusted temperature profiles indicate that the mean measured and computed radiation intensities agree to within $10-20 \%$ (depending on the spectral band) if the mean measured and computed temperature distributions agree. These calculations indicate that models within the LES cause $20-30 \%$ of the difference between the measured and computed radiation intensities, and a combination of the narrowband radiation model and experimental uncertainty cause $10-20 \%$ of the difference. The quantitative similarities and differences between the measured and computed radiation intensities demonstrate one of the significant benefits of the model-based mid-infrared imaging approach: the ability to capture subtle differences in scalar values and to highlight opportunities for model improvement by using the non-linear relationships between radiation intensities and the species concentrations and temperature distributions as an advantage.

The model-based mid-infrared imaging approach also provides the unique capability to observe the flame from multiple observer positions at the same instant in time. Instantaneous images are computed of the radiation intensity as observed from multiple angles orthogonal to the flame axis. The change in camera observer angle is accomplished numerically by rotating the LES scalar results about the flame axis in the azimuthal direction by an angular increment 
consistent with the angular spatial resolution of the LES results. Figure 6 shows computed images of the mid-infrared radiation intensity from the turbulent nonpremixed flame as observed from multiple angles orthogonal to the flame axis at one instant in time. The increment in the observer angle between images shown here is $45^{\circ}$ in the azimuthal direction. Significant variation in the instantaneous radiation intensity is apparent in the images when the flame is observed from different positions. Significant spatial correlation in the instantaneous radiation intensity is evident when the flame is observed from positions separated by approximately $5.6^{\circ}$ (figures not reported here in the interest of brevity), consistent with the spatial resolution of LES results. The images separated by $180^{\circ}$ are approximately mirror images of one another, and the radiation emitted by the flame is qualitatively and quantitatively similar when observed from opposite sides of the flame. Subtle differences in the images from the two angles separated by $180^{\circ}$ are indicative of the small influence of radiation absorption effects for the spectral bands considered in this work.

\section{Conclusions}

Quantitative model-based images of the radiation intensity from a turbulent nonpremixed flame are rendered in this work. These results offer benchmark data that are useful for assessing LES combustion models and identifying model-sensitivities such as the effects of radiation and buoyancy. Specific conclusions from the qualitative and quantitative comparison of the measured and computed images of the radiation intensity include the following:

(1) The measured and computed images of the radiation intensity from the turbulent nonpremixed flame display similar qualitative features including localized regions of high and low intensity characteristic of turbulent mixing between the fuel jet, entrained air, and 
combustion products. Qualitative similarities between the measurements and computations demonstrate the potential of utilizing model-based imaging of radiation intensity for evaluation of radiation and combustion models.

(2) Including radiation heat loss is important even for weakly radiating flames with low radiant heat loss fractions (e.g. less than 10\%), particularly in the region downstream of the stoichiometric flame length.

(3) The consistency between conclusions based on path-integrated radiation intensities and scalar distributions demonstrates that the present model-based image rendering approach is useful for improving understanding and evaluating radiation and combustion models. The present approach of quantitatively comparing radiation intensities is a useful complementary strategy to the conventional approach of analyzing scalar flow-field results.

\section{Acknowledgements}

The authors thank Dr. Robert Barlow and Dr. Gaetano Magnotti for collaborating on the radiation intensity measurements of the turbulent nonpremixed flame acquired at the Sandia National Laboratories Combustion Research Facility. Computational simulations were performed using resources of the National Energy Research Scientific Computing Center, which is supported by the Office of Science of the U.S. Department of Energy under Contract No. DEAC02-05CH11231.

\section{References}

[1] J.H. Frank, R.S. Barlow, C. Lundquist, Proceedings of the Combustion Institute, 28 (2000) 447-454.

[2] Y. Zheng, Y.R. Sivathanu, J.P. Gore, Proceedings of the Combustion Institute, 29 (2002) 1957-1963.

[3] Y. Zheng, R.S. Barlow, J.P. Gore, Journal of Heat Transfer, 125 (2003) 678-686.

[4] M.R. Roomina, R.W. Bilger, Combustion and Flame, 125 (2001) 1176-1195.

[5] G. Li, M.F. Modest, Journal of Heat Transfer, 125 (2003) 831-838. 
[6] G. Li, M.F. Modest, Journal of Quantitative Spectroscopy \& Radiative Transfer, 73 (2002) 461 -472.

[7] A. Wang, M.F. Modest, D.C. Haworth, L. Wang, Journal of Quantitative Spectroscopy \& Radiative Transfer, 109 (2008) 269-279.

[8] M. Ihme, H. Pitsch, Physics of Fluids, 20 (2008) 055110.

[9] P.J. Coelho, Combustion and Flame, 156 (2009) 1099-1110.

[10] A. Gupta, D.C. Haworth, M.F. Modest, Proceedings of the Combustion Institute, 34 (2013) 1281-1288.

[11] M. Carvalho, T. Farias, Chemical Engineering Research and Design, 76 (1998) 175-184.

[12] W. Malalasekera, H.K. Versteeg, J.C. Henson, J.C. Jones, Clean Air, 3 (2002) 113-143.

[13] M.F. Modest, International Journal for Multiscale Computational Engineering, 3 (2005) 85-105.

[14] P.J. Coelho, Progress in Energy and Combustion Science, 33 (2007) 311-383.

[15] R. Viskanta, International Journal of Thermal Sciences, 47 (2008) 1563-1570.

[16] P.J. Coelho, Journal of Heat Transfer, 134 (2012) 031001.

[17] B.A. Rankin, D.L. Blunck, V.R. Katta, S.D. Stouffer, J.P. Gore, Combustion and Flame, 159 (2012) 2841 2843.

[18] B.A. Rankin, Purdue University, West Lafayette, IN, 2012.

[19] B.A. Rankin, D.L. Blunck, J.P. Gore, Journal of Heat Transfer, 135 (2013) 021201.

[20] B.A. Rankin, G. Magnotti, R.S. Barlow, J.P. Gore, Combustion and Flame, (2014) in press.

[21] International Workshop on Measurement and Computation of Turbulent Nonpremixed Flames, http://www.sandia.gov/TNF/.

[22] G. Havener, 18th AIAA Aerospace Ground Testing Conference, Colorado Springs, CO, 1994.

[23] M.G. Mungal, A. Lozano, I. Vancruyningen, Experiments in Fluids, 12 (1992) 141-150.

[24] M.G. Mungal, A. Lozano, Experiments in Fluids, 21 (1996) 257-264.

[25] H. Yu, C. Wang, R.W. Grout, J.H. Chen, K.-L. Ma, IEEE Computer Graphics and Applications, (2010) 45-57.

[26] A. Tikhonova, H. Yu, C.D. Correa, J.H. Chen, K.-L. Ma, Eurographics Symposium on Parallel Graphics and Visualization, (2011) 1-11.

[27] G.P. Forney, D. Madrzykowski, K.B. McGrattan, IEEE Computer Graphics and Applications, (2003) 6-13.

[28] W. Roquemore, V. Katta, S. Stouffer, V. Belovich, R. Pawlik, M. Arstingstall, G. Justinger, J. Gord, A. Lynch, J. Zelina, Proceedings of the Combustion Institute, 32 (2009) 729-736.

[29] B. Connelly, B. Bennett, M. Smooke, M. Long, Proceedings of the Combustion Institute, 32 (2009) 879-886.

[30] M. Roger, C.B. Da Silva, P.J. Coelho, International Journal of Heat and Mass Transfer, 52 (2009) 2243 -2254.

[31] M. Roger, P.J. Coelho, C.B. da Silva, International Journal of Heat and Mass Transfer, 53 (2010) 2897-2907.

[32] D. Poitou, J. Amaya, M. El Hafi, B. Cuénot, Combustion and Flame, 159 (2012) 1605-1618.

[33] W. Bergmann, W. Meier, D. Wolff, W. Stricker, Applied Physics B, 66 (1998) 489-502.

[34] W. Meier, R.S. Barlow, Y.L. Chen, J.Y. Chen, Combustion and Flame, 123 (2000) 326-343.

[35] C. Schneider, A. Dreizler, J. Janicka, E.P. Hassel, Combustion and Flame, 135 (2003) 185-190.

[36] M. Ihme, H. Pitsch, International Journal of Aeroacoustics, 11 (2012) 25-78.

[37] M. Ihme, Y.C. See, Combustion and Flame, 157 (2010) 1850-1862.

[38] M. Ihme, L. Shunn, J. Zhang, Journal of Computational Physics, 231 (2012) 7715-7721.

[39] J.P. Gore, J.H. Jang, Journal of Heat Transfer, 114 (1992) 234-242.

[40] R.S. Barlow, A.N. Karpetis, J.H. Frank, J.Y. Chen, Combustion and Flame, 127 (2001) 2102-2118.

[41] W.L. Grosshandler, National Institute of Standards and Technology, Washington, D.C., 1993.

[42] J.P. Gore, U.S. Ip, Y.R. Sivathanu, Journal of Heat Transfer, 114 (1992) 487-493. 
Figure 1. Schematic of the experimental arrangement and discrete domain for rendering measured and computed results of the mid-infrared radiation intensity emitted from the turbulent nonpremixed flame. Note: $(x, r, \theta)$ are flame-based (observed) cylindrical coordinates, $(x, y, z)$ are flame-based (observed) Cartesian coordinates, and $(X, Y, R)$ are camera-based (observer) Cartesian coordinates.

Figure 2. Measured and computed mean and root mean square temperature (left), $\mathrm{CO}_{2}$ mole fraction (middle), and $\mathrm{H}_{2} \mathrm{O}$ mole fraction (right) profiles along the centerline of the flame.

Figure 3. Measured and computed radial profiles at selected axial locations of mean and root mean square temperature (left), $\mathrm{CO}_{2}$ mole fraction (middle), and $\mathrm{H}_{2} \mathrm{O}$ mole fraction (right).

Figure 4. Time-dependent and time-averaged images of the mid-infrared radiation intensity $(2.77 \pm 0.12 \mu \mathrm{m})$ from the turbulent nonpremixed flame (DLR-A) for the measurements [(a) (c)], adiabatic LES [(d) - (f)], LES including radiation [(g) - (i)], and LES including radiation and buoyancy $[(j)-(k)]$. The horizontal white lines denote the field of view associated with the measured images.

Figure 5. Measured and computed time-averaged mid-infrared radiation intensities from diametric paths through the turbulent nonpremixed flame (DLR-A).

Figure 6. Computed images of the mid-infrared radiation intensity $(2.77 \pm 0.12 \mu \mathrm{m})$ from the turbulent nonpremixed flame (DLR-A) as observed at one instant in time from multiple angles $\left(0^{\circ}-180^{\circ}\right.$ in increments of $\left.45^{\circ}\right)$ orthogonal to the flame axis. The computed images are based on adiabatic LES results.

Table 1. Measured and computed peak mean radiation intensities $\left(\mathrm{W} / \mathrm{m}^{2}-\mathrm{sr}\right)$ for the three spectral bands and the relative difference (\%) between the measured and computed values for diametric paths near the $X / D=60$ location (which has been selected only for illustration purposes).

\begin{tabular}{|l|c|c|c|c|c|c|}
\hline & \multicolumn{2}{|c|}{$2.58 \pm 0.03 \mu \mathrm{m}$} & \multicolumn{2}{c|}{$2.77 \pm 0.12 \mu \mathrm{m}$} & \multicolumn{2}{c|}{$4.34 \pm 0.10 \mu \mathrm{m}$} \\
\hline & $\begin{array}{c}\text { Intensity } \\
\left(\mathrm{W} / \mathrm{m}^{2}-\mathrm{sr}\right)\end{array}$ & $\begin{array}{c}\text { Difference } \\
(\%)\end{array}$ & $\begin{array}{c}\text { Intensity } \\
\left(\mathrm{W} / \mathrm{m}^{2}-\mathrm{sr}\right)\end{array}$ & $\begin{array}{c}\text { Difference } \\
(\%)\end{array}$ & $\begin{array}{c}\text { Intensity } \\
\left(\mathrm{W} / \mathrm{m}^{2}-\mathrm{sr}\right)\end{array}$ & $\begin{array}{c}\text { Difference } \\
(\%)\end{array}$ \\
\hline Measured (Camera) & 75 & - & 340 & - & 910 & - \\
\hline Adiabatic & 110 & 45 & 540 & 60 & 1330 & 45 \\
\hline Radiation & 100 & 35 & 490 & 45 & 1270 & 40 \\
\hline $\begin{array}{l}\text { Radiation + } \\
\text { Buoyancy }\end{array}$ & 100 & 35 & 490 & 45 & 1270 & 40 \\
\hline $\begin{array}{l}\text { Simulated } \\
\text { (Corrected Scalars) }\end{array}$ & 85 & 15 & 410 & 20 & 990 & 10 \\
\hline
\end{tabular}


Figure1

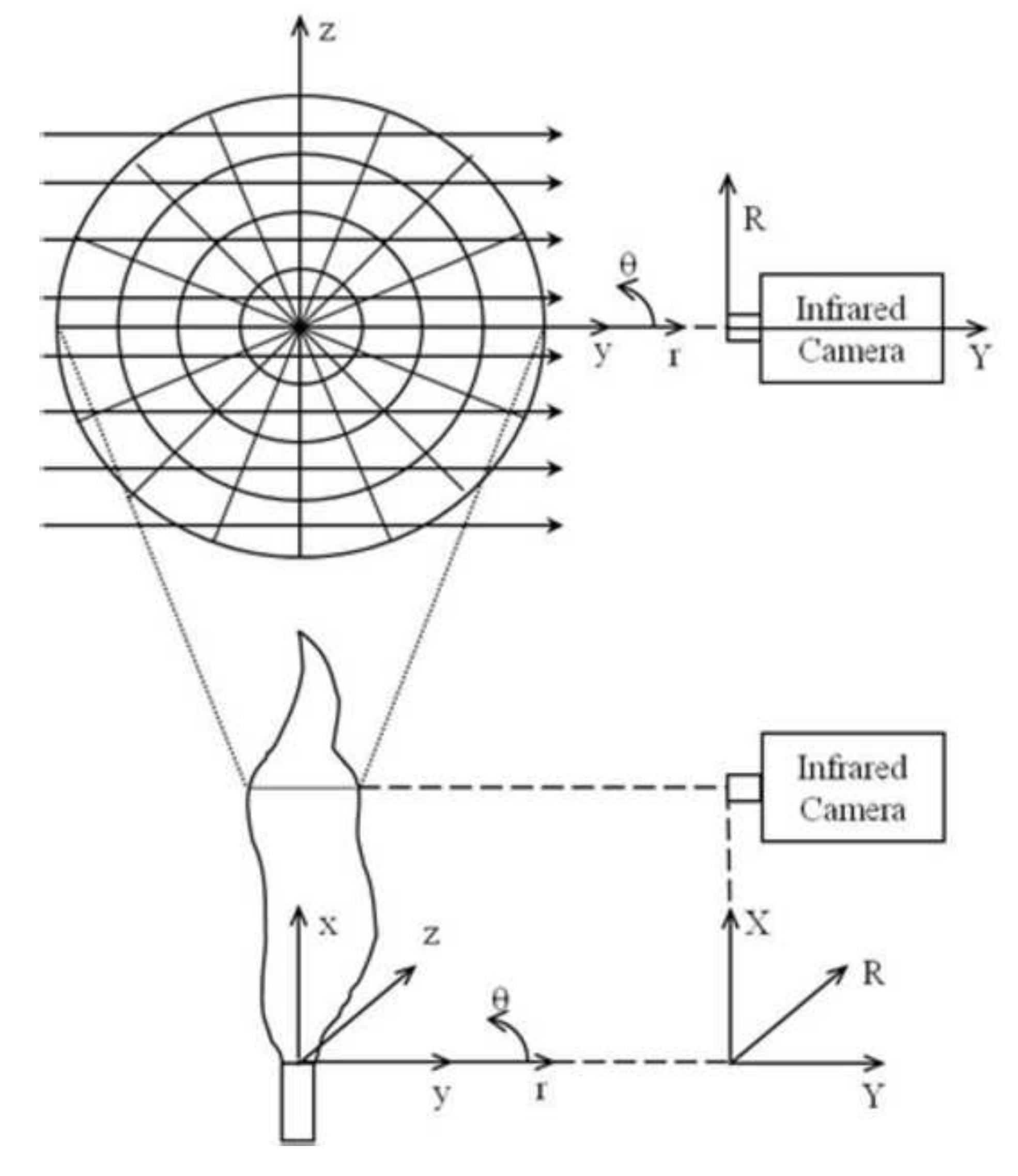

Figures

s
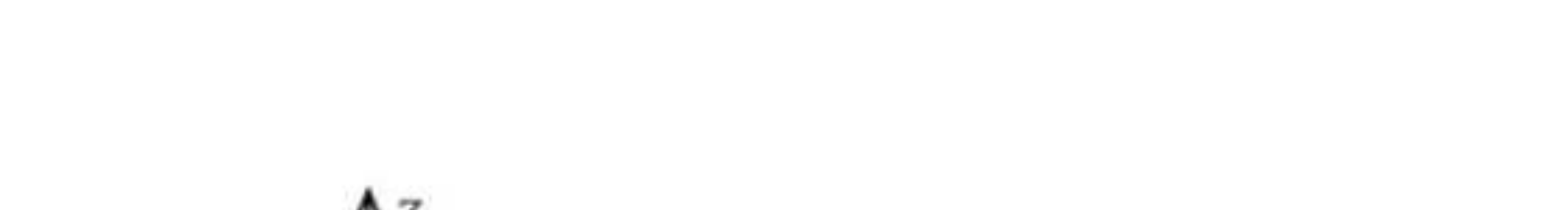

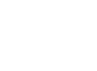
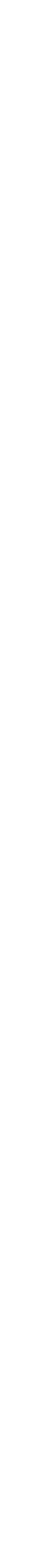

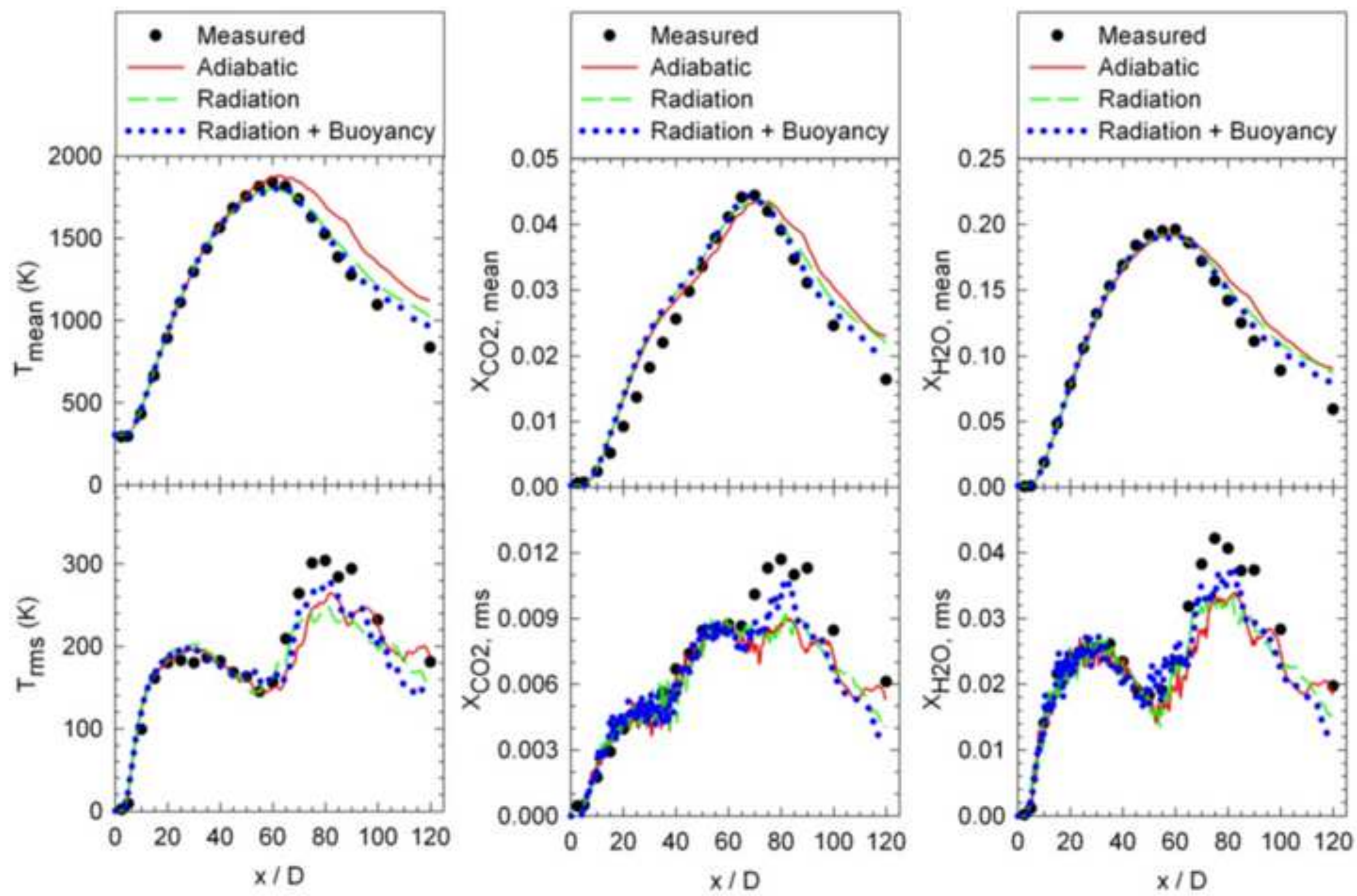

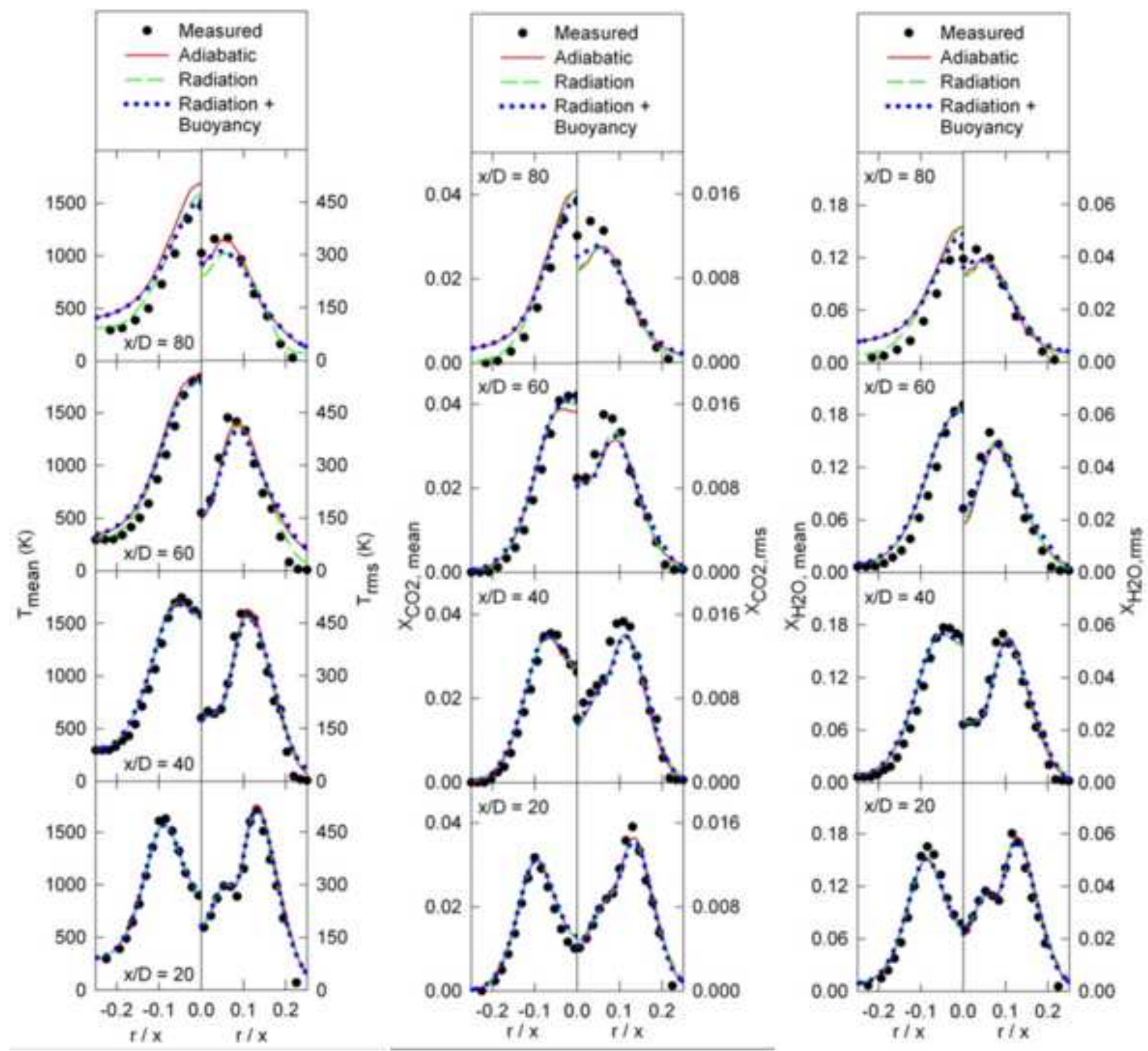

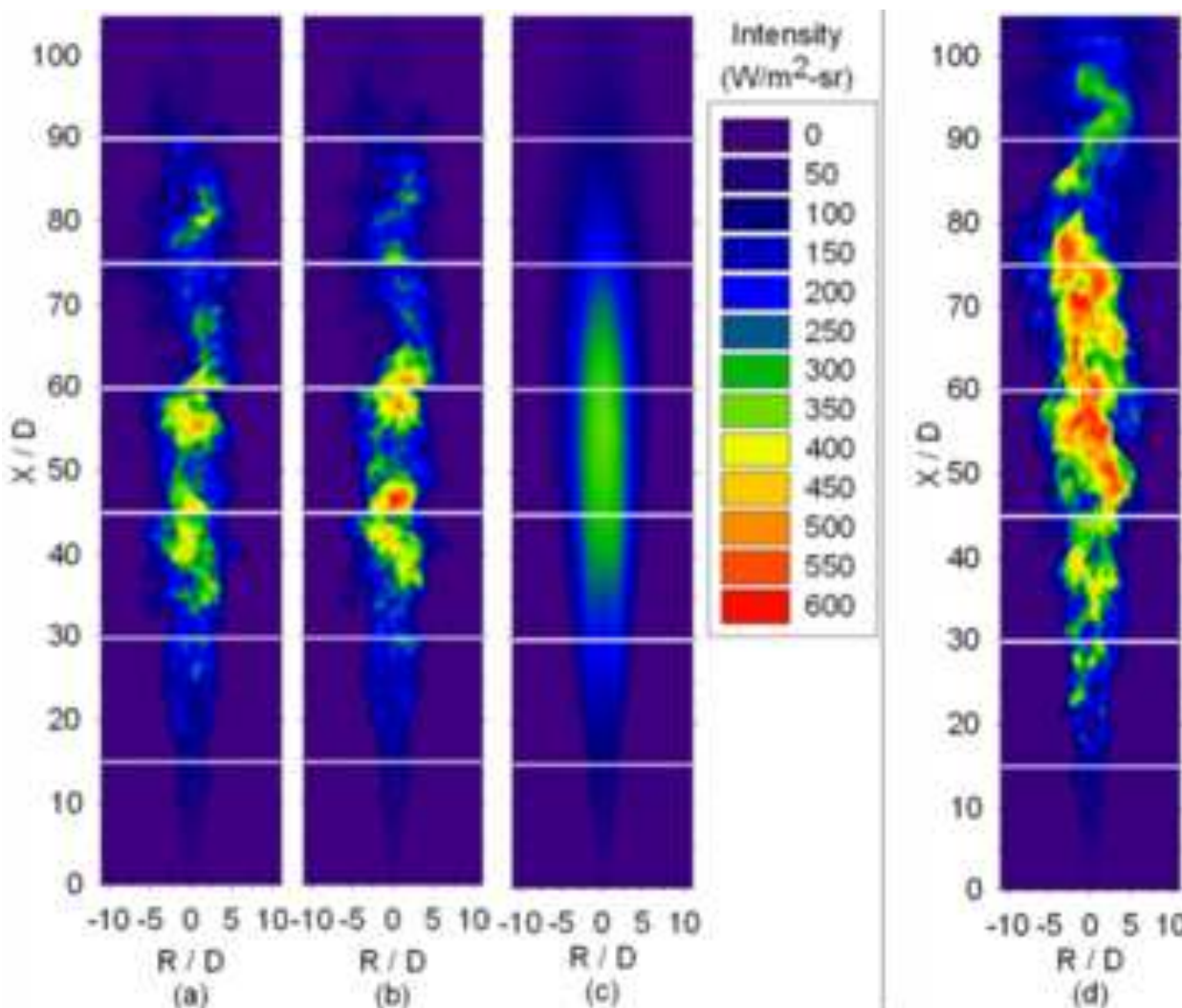

(d)

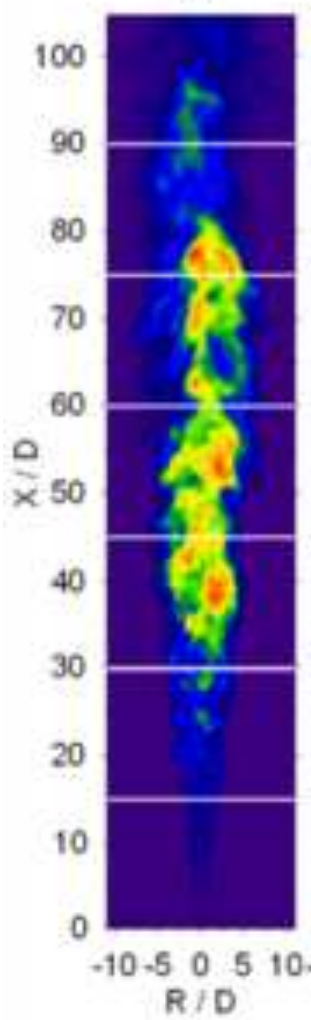

(g)
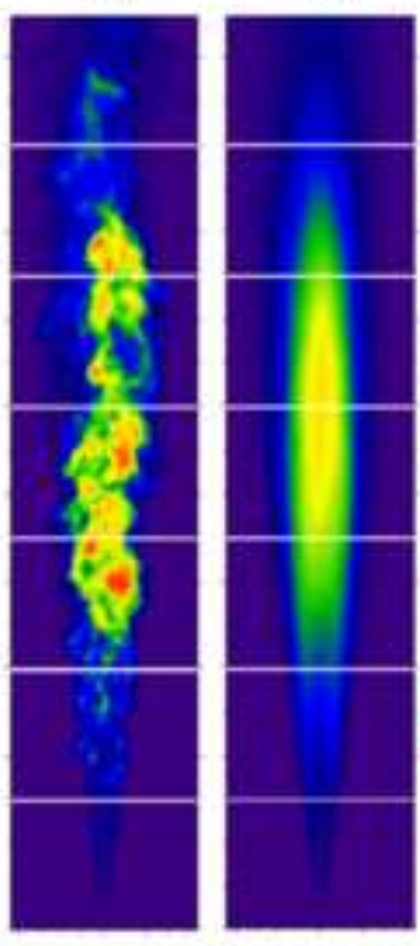

Intensity (Wim²sr)
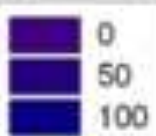
150 200 260 300 350 400 450 500 550 600 650 700

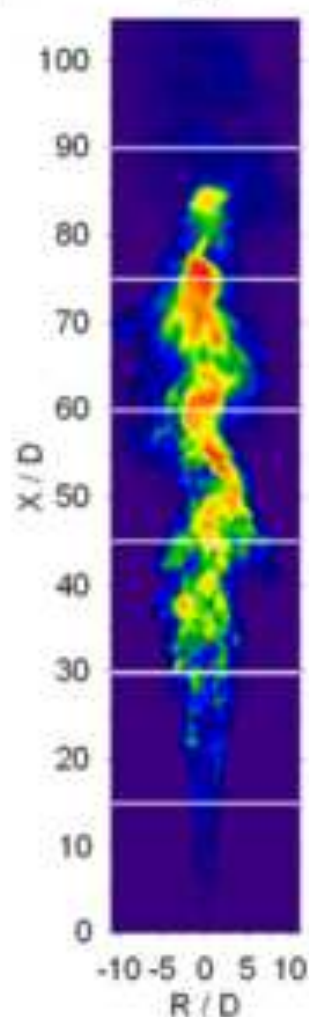

(i)
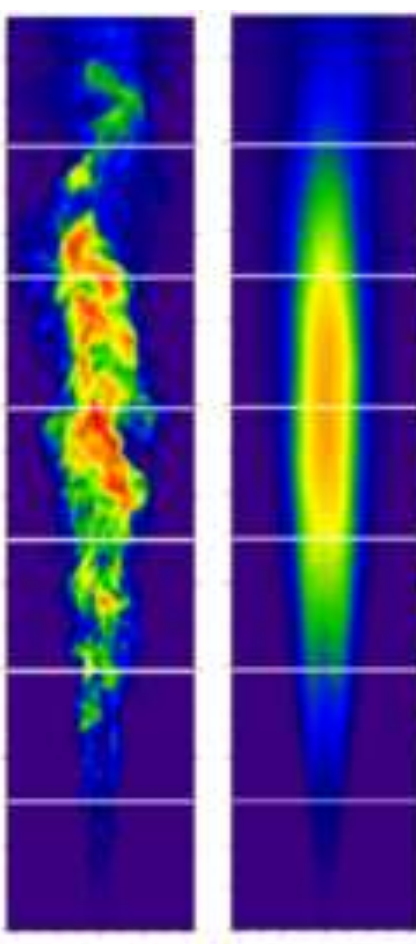

$\mathrm{R} / \mathrm{D}$

(e)
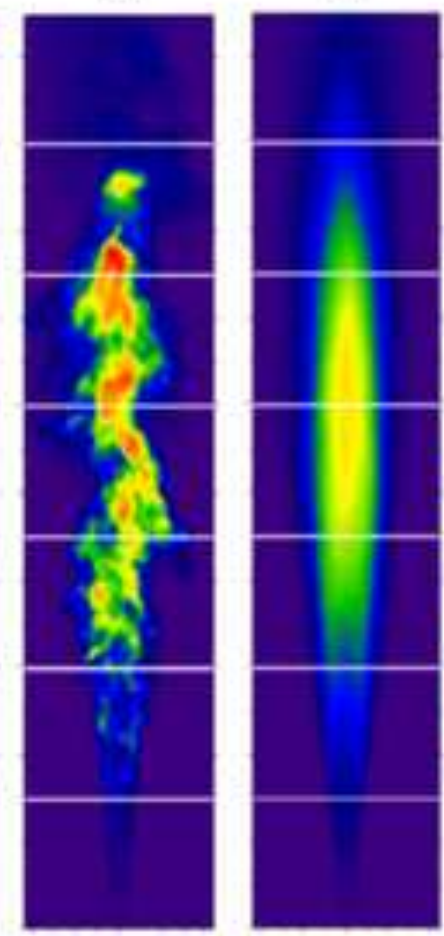

(f)

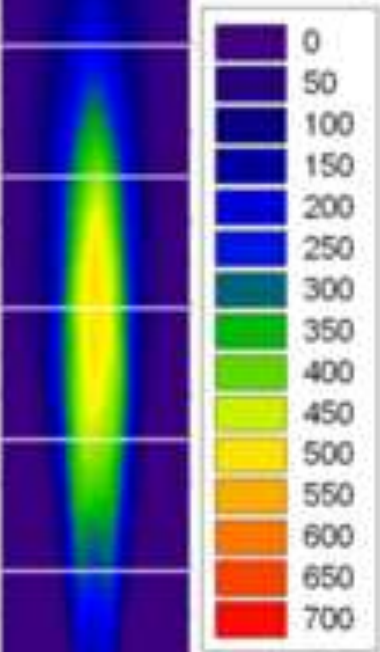

Intensity $\left(W^{2} m^{2}-5 n\right)$ $\left(W m^{2}-s\right)$

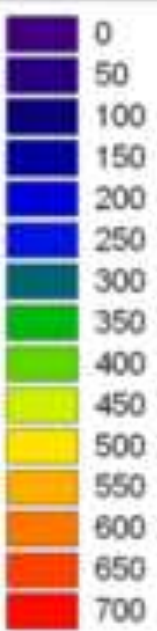

Intensity

700

\section{(}




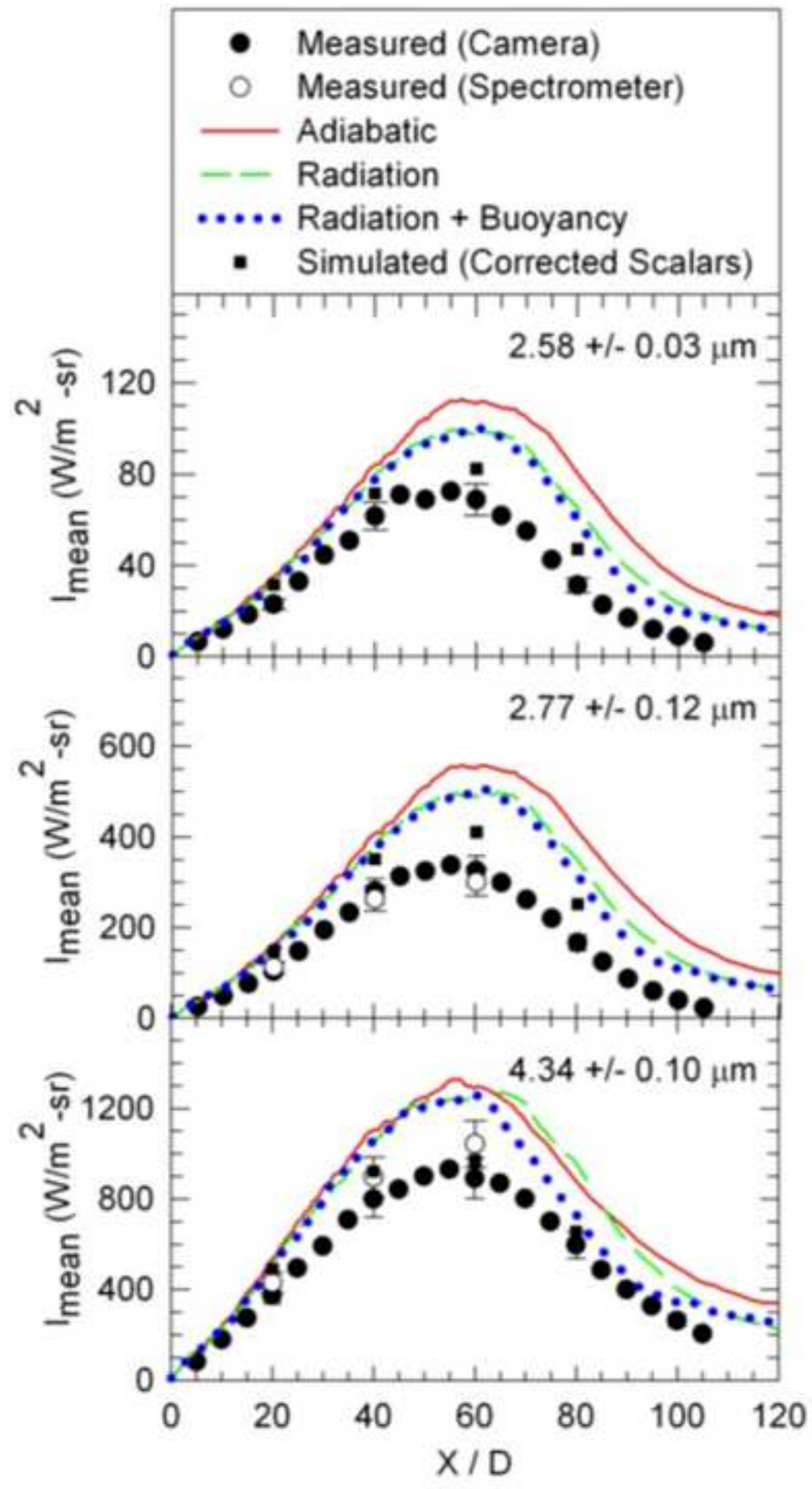

- Measured (Camera)

Adiabatic

- Simulated (Corrected Scalars) 


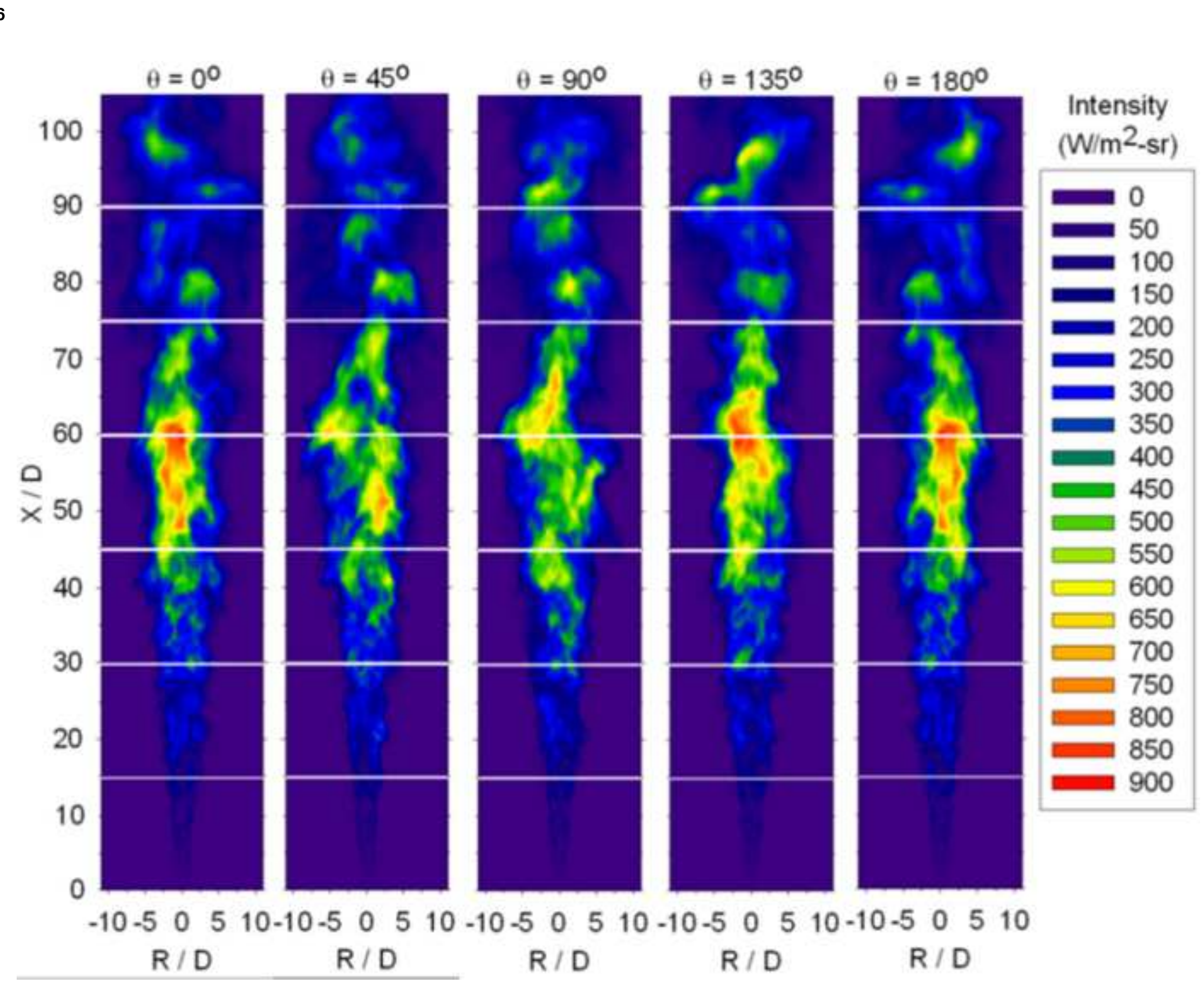

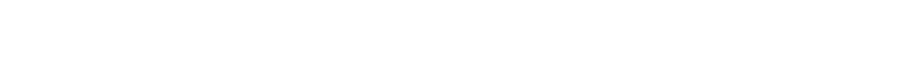

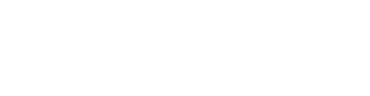$$
\text { (2n) }
$$

.

